\title{
Two-phase multi-model automatic brain tumour diagnosis system from magnetic resonance images using convolutional neural networks
}

\author{
Mahmoud Khaled Abd-Ellah', Ali Ismail Awad ${ }^{2,3^{*}}$ (D), Ashraf A. M. Khalaf ${ }^{4}$ and Hesham F. A. Hamed ${ }^{4}$
}

\begin{abstract}
Brain tumour is a serious disease, and the number of people who are dying due to brain tumours is increasing. Manual tumour diagnosis from magnetic resonance images (MRIs) is a time consuming process and is insufficient for accurately detecting, localizing, and classifying the tumour type. This research proposes a novel two-phase multi-model automatic diagnosis system for brain tumour detection and localization. In the first phase, the system structure consists of preprocessing, feature extraction using a convolutional neural network (CNN), and feature classification using the error-correcting output codes support vector machine (ECOC-SVM) approach. The purpose of the first system phase is to detect brain tumour by classifying the MRIs into normal and abnormal images. The aim of the second system phase is to localize the tumour within the abnormal MRIs using a fully designed five-layer region-based convolutional neural network (R-CNN). The performance of the first phase was assessed using three CNN models, namely, AlexNet, Visual Geometry Group (VGG)-16, and VGG-19, and a maximum detection accuracy of 99.55\% was achieved with AlexNet using 349 images extracted from the standard Reference Image Database to Evaluate Response (RIDER) Neuro MRI database. The brain tumour localization phase was evaluated using 804 3D MRIs from the Brain Tumor Segmentation (BraTS) 2013 database, and a DICE score of 0.87 was achieved. The empirical work proved the outstanding performance of the proposed deep learning-based system in tumour detection compared to other non-deep-learning approaches in the literature. The obtained results also demonstrate the superiority of the proposed system concerning both tumour detection and localization.
\end{abstract}

Keywords: Brain tumour diagnosis, MRI segmentation, Tumour detection and localization, Convolutional neural networks (CNNs)

\section{Introduction}

Brain tumour is a serious disease in which an abnormal growth of tissue inside the the brain can disrupt proper brain function. The National Brain Tumor Foundation (NBTF) reported that the number of people in developed countries who have died due to brain tumours has increased by $300 \%$ over the last three decades $[1,2]$. Manual brain tumour diagnosis by physicians is a less accurate yet still time-consuming procedure $[3,4]$.

\footnotetext{
${ }^{*}$ Correspondence: ali.awad@|tu.se

${ }^{2}$ Department of Computer Science, Electrical and Space Engineering, Luleå

University of Technology, SE-97187 Luleå, Sweden

${ }^{3}$ Faculty of Engineering, Al-Azhar University, P.O. Box 83513, Qena, Egypt

Full list of author information is available at the end of the article
}

Magnetic resonance imaging is an advanced medical imaging technique providing rich information about the human soft tissue anatomy [5]. Automatic brain tumour detection from magnetic resonance images (MRIs) aims to classify MRIs into normal and abnormal according to the absence or presence of a tumour, respectively. Thus, the diagnosis problem can be addressed as an image classification challenge. The pervasiveness of brain tumours has led to the production of a massive amount of MRI data. Therefore, developing an automatic brain tumour diagnosis system that achieves high tumour detection and localization accuracies is a vital need [6]. Machine learning in general and deep learning approaches in particular play core roles in computer-assisted brain image analysis, 
segmentation, registration, and tumour tissue classification $[7,8]$.

Recently, deep learning paradigms have received considerable research attention. Deep learning models have multiple layers that can be trained using either supervised or unsupervised training approaches $[9,10]$. In recent studies, deep learning models have achieved competitive results compared to the classical learning models in brain tumour diagnosis and tumour image classification $[11,12]$. In addition, deep learning models like convolutional neural networks (CNNs) have improved the field of object detection and classification in different applications $[13,14]$. As a deep learning paradigm, CNNs [15] have been used to extract high-level features from raw image data [16].

Although several studies have targeted the application of deep learning methods to brain tumour diagnosis, a complete system for automatic tumour detection and localization is not yet available in the literature. In addition, integrating tumour detection and localization accuracy within a single diagnosis system is still an open challenge. Having brain tumour detection phase preceding the tumour localization in a single system leads to discarding the normal images from being processed in the localization phase. This opens doors to the real-time deployment of such automatic tumour diagnosis systems that save time and computing power from locating tumours in normal images.

This research addresses the aforementioned challenges by developing a novel and complete computer-aided diagnosis (CAD) system for tumour detection and localization from MRIs. In the tumour detection phase, the system combines a $\mathrm{CNN}$, which is used for feature extraction due to its ability to learn features from raw data, with an error-correcting output codes support vector machine (ECOC-SVM), which is used for feature classification. The system is considered a two-phase multi-model artefact due to its detection and localization abilities using different CNN models.

Three CNN models, namely, AlexNet, Visual Geometry Group (VGG)-16, and VGG-19, have been deployed and evaluated for tumour detection. A five-layer fully developed region-based convolutional neural network ( $\mathrm{R}$ $\mathrm{CNN}$ ) has been used in the second phase for tumour localization. The accuracy of the developed two-phase multi-model system has been evaluated using MRIs extracted from two different databases: the Reference Image Database to Evaluate Response (RIDER) Neuro MRI database [17] and the Brain Tumor Segmentation (BraTS) 2013 database.

The key contributions of this study exist in several dimensions. First, a complete two-phase multi-model system for brain tumour detection and localization that combines a CNN and an ECOC-SVM is implemented and evaluated. Although CNNs and SVMs are not new approaches, the state-of-the-art in this field does not yet include systems similar to the one developed here, i.e., with these specific phases and CNN learning models. Second, integrating tumour detection and localization in one artefact is a good step towards real-time deployment of the research findings. Third, 349 MRIs from the RIDER Neuro MRI dataset are extracted and manually annotated, and this dataset will be available as a standard benchmark for other researchers who conduct research on tumour detection. Fourth, the conducted experimental works have proven the superiority of the developed system in terms of tumour detection and tumour localization.

The remainder of this article is organized as follows. Section 2 is devoted to reporting related work on both the detection and localization of brain tumours. The description of the two-phase multi-model tumour diagnosis system with respect to design and implementation is provided in Section 3. The experimental studies and the simulations for evaluating the whole diagnosis system in terms of tumour detection and localization accuracy are documented in Section 4. Finally, concluding remarks are outlined in Section 5.

\section{Related work}

Brain tumour detection has received substantial research attention, and several detection methods have been presented over the past two decades. In [18], a combination of morphological filters, discrete wavelet transforms (DWTs), principal component analysis (PCA), and kernel support vector machines (KSVM) were applied to classify MRIs as normal and abnormal. Further improvements have been realized to classify an abnormal image as benign (noncancerous) or malignant (cancerous) by applying a double classifier Abd-Ellah et al. [19]. Devasena and Hemalatha [20] proposed a CAD system for the detection of abnormal parts in MRIs using a hybrid abnormality detection algorithm (HADA).

In [21], Patil and Udupi used preprocessing, segmentation, feature extraction, and a probabilistic neural network (PNN) method to identify brain tumours. Arakeri and Reddy [22] applied three different classifiers: an SVM, an artificial neural network (ANN), and a $k$-nearest neighbour ( $\mathrm{k}-\mathrm{NN}$ ) algorithm to distinguish between different types of tumours. Dandil et al. [23] proposed a system for brain tumour classification where spatial-fuzzy C-means (FCM) was applied for brain tumour segmentation, and an SVM was used in the classification stage.

Goswami and Bhaiya [24] presented a new MRI brain tumour classification based on an ANN. Histogram equalization, noise filtering, and edge detection were applied in the preprocessing stage. The features were extracted by independent component analysis (ICA). A self-organizing map neural network (SOMNN) was 
applied in the classification stage. Deepa and Devi [25] proposed a system consisting of feature extraction, classification, and tumour segmentation. Optimal texture features were extracted from tested images using statistical features. A back propagation neural network (BPN) and a radial basis function neural network (RBFN) were used in the classification and segmentation stages, respectively. A short comparison of the aforementioned classical learning-based diagnosis methods is provided in Table 1.

Few deep learning-based methods for brain tumour diagnosis were found in the state-of-the-art in this field. In [26], brain CT image classification based on a deep neural network (DNN) was presented. A gray-level cooccurrence matrix (GLCM) was used for feature extraction, and a DNN was applied for classification. The average accuracy of the reported method was $83 \%$. Gao et al. [27] introduced a CT brain image classification that integrated both 2D and 3D CNNs. The average classification accuracy rate was $87.6 \%$, while each network consisted of seven layers. Yan $\mathrm{Xu}$ et al. proposed a method using deep convolutional activation features for brain tumour classification and segmentation. The system classification accuracy was $97.5 \%$ [28].

Several deep learning methods for brain tumour localization have been presented in the literature. Havaei et al. [29] demonstrated automatic brain tumour localization using a DNN based on the CNNs with an average DICE score of 0.88 .

In [30], a fully convolutional residual neural network (FCR-NN) combined with a fully convolutional architecture with optimization gains from residual identity was developed. The network consists of a 22 complex layers, and the achieved DICE score was 0.87. Casamitjana et al.

Table 1 Comparison of the available traditional classification approaches used in brain tumour detection

\begin{tabular}{llll}
\hline CAD system & Total images & $\begin{array}{l}\text { Classification } \\
\text { method }\end{array}$ & Accuracy (\%) \\
\hline $\begin{array}{l}\text { El-Dahshan } \\
\text { et al. [2] }\end{array}$ & 101 & FFBPNN & 99.00 \\
$\begin{array}{l}\text { Devasena and } \\
\text { Hemalatha [20] }\end{array}$ & 250 & HADA & 98.8 \\
$\begin{array}{l}\text { Arakeri and } \\
\text { Reddy [22] }\end{array}$ & 550 & $\begin{array}{l}\text { SVM, ANN and } \\
\text { k-NN }\end{array}$ & 99.09 \\
$\begin{array}{l}\text { Dandıl et al. [23] } \\
\text { Goswami and }\end{array}$ & 70 & KSVM & 91.49 \\
$\begin{array}{l}\text { Bhaiya } \\
\text { [24] }\end{array}$ & & SOMNN & 98.60 \\
$\begin{array}{l}\text { Deepa and } \\
\text { Devi [25] }\end{array}$ & 42 & BPN and RBFN & 85.71 \\
$\begin{array}{l}\text { Abd-Ellah } \\
\text { et al. [18] }\end{array}$ & 80 & KSVM & 100 \\
$\begin{array}{l}\text { Abd-Ellah } \\
\text { et al. [19] }\end{array}$ & 120 & KSVM & 100 \\
\hline
\end{tabular}

[31] proposed the use of $3 \mathrm{D}$ CNNs for the segmentation of abnormal parts in MRIs based on a network using multi-resolution features and reported a DICE score of 0.84 .

Zhao et al. [32] used a fully convolutional neural network (FCNN) and conditional random fields (CRFs) as a post-processing step to segment brain tumours with an obtained DICE score of 0.87. Pereira et al. in [33] presented a preliminary study on brain tumour segmentation based on fully convolutional network (FCN) with standardization of MRI histograms as a preprocessing stage, which demonstrated a DICE score of 0.85 . More recently, Pereira et al. [34] examined a complete segmentation system that includes preprocessing, $\mathrm{CNN}$, and post-processing stages. The new system could achieve an improved DICE score of 0.88 at the expense of increased system complexity.

\section{Methods}

Currently, deep learning models are of considerable research importance. Deep learning methods provide high efficiency and the ability to process numerous MRIs from databases [35]. This study focuses on CNNs, which have gained popularity among researchers for object recognition and biological image segmentation.

The developed diagnosis system has two phases for tumour detection and localization. The first phase converts tumour detection into an MRI classification problem and then categorizes MRIs into normal and abnormal images. The second phase focuses on tumour localization inside the abnormal images. Figure 1 shows a block diagram of the proposed system. MRIs are delivered to the system's input, and then, the preprocessing tasks are performed. A feature extraction tool is used to select the relevant features, and a classification tool is applied to decide whether these features belong to a normal or an abnormal MRI. To achieve accurate results, the fivelayer R-CNN has been trained and evaluated using MRIs from BraTS 2013 as a standard benchmark for tumour localization.

\subsection{Brain tumour detection phase \\ 3.1.1 Input image and preprocessing}

Due to the ability of magnetic resonance imaging to provide information about the soft tissues in the brain, databases of MRIs were used in this study as an input to the developed system. The goal of the preprocessing stage is to ensure the MRI readiness for the next stage. It improves image quality, reduces the noise level, and enhances the overall image resolution by employing classical filtering techniques [36]. The preprocessing stage also adjusts the image size and type to a $227 \times 227 \mathrm{RGB}$ image, which is provided to the trainable $\mathrm{CNN}$ in the feature extraction stage. 


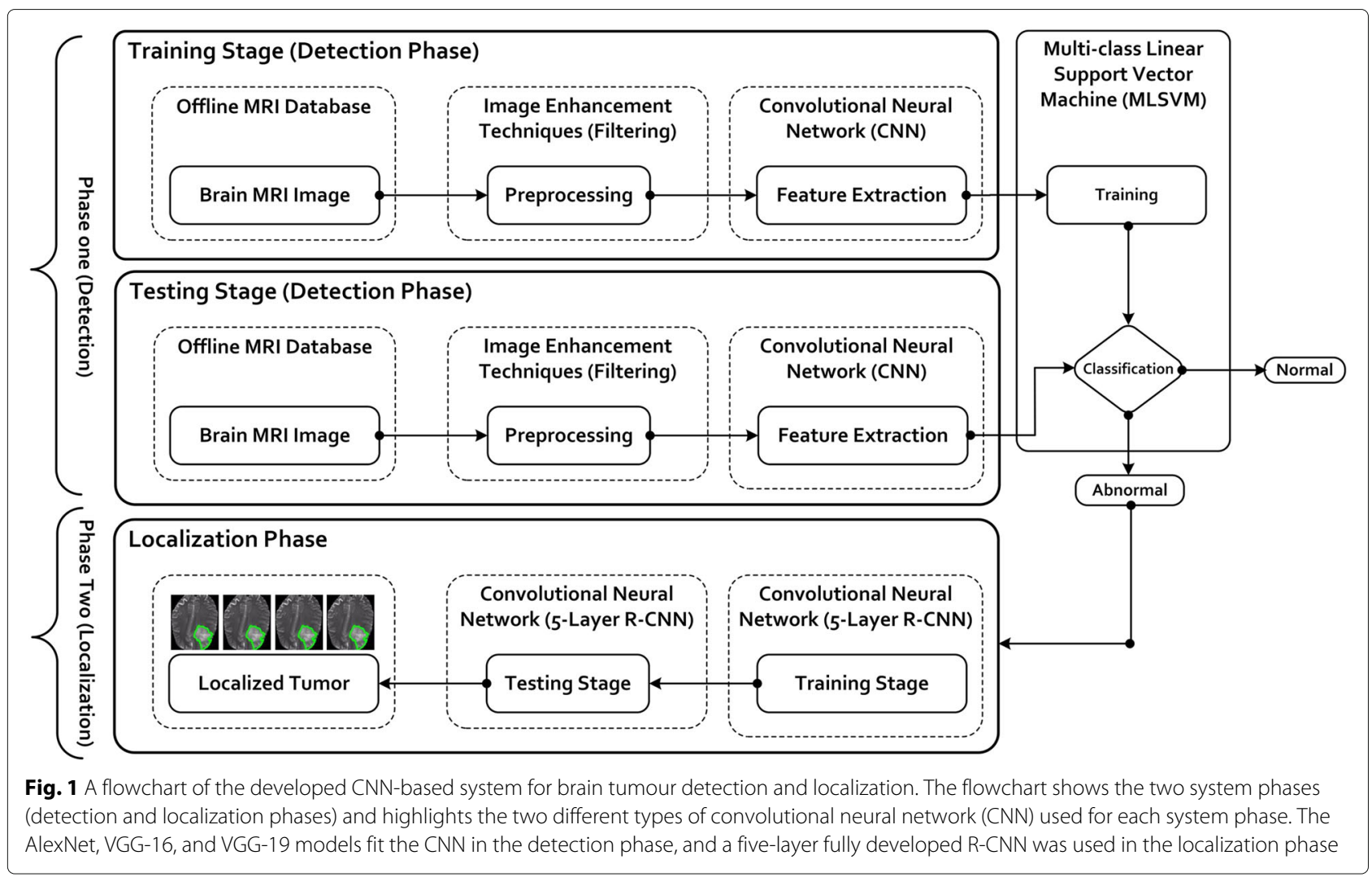

\subsubsection{Convolutional neural network (CNN)}

$\mathrm{CNN}$ is the deep learning model that has improved over the past two decades. CNNs can learn features automatically from input data, especially images, as is the case in this research [37]. The convolutional layers are used to convolve the input image with kernels (weights) to obtain a feature map. The weights of the kernels connect the feature map units to the previous layer. AlexNet and two other CNN models, namely, VGG-16 and VGG-19, were used and evaluated in the tumour detection phase. However, the focus of the theoretical foundation section is on the AlexNet model. The AlexNet model was selected due to its flexibility to be modified, its ability to reduce overfitting using a dropout layer, and its capability to train faster through using a rectified linear unit (ReLU).

The AlexNet model utilized for tumour detection is a pre-trained network from the MatConvNet toolbox [12]. It consists of 23 layers with weights. The first layer defines the input dimensions, including an input image size of $227 \times 227 \times 3$. A series of convolutional layers intervene with ReLU and max-pooling layers. The final layer is a classification layer with 1000 classes.

The tumour detection phase employs the $\mathrm{CNN}$ network for feature extraction, and there are few layers in the $\mathrm{CNN}$ that are suitable for this goal. The CNN depends on nine layers, as shown in Fig. 2: an image input, two convolution, two ReLU, two cross channel normalization (Norm), and two max-pooling layers. Layers 2 and 6 are convolutional layers with $11 \times 11 \times 3$ and $5 \times 5 \times 48$ convolutions, respectively. If we suppose that the input $x$ and the weight $w$ are realized on integer $t$, the $2 \mathrm{D}$ discrete convolution can be defined as in Eq. 1:

$$
y(i, j)=x(i, j) * w(i, j)=\sum_{m} \sum_{n} x(m, n) w(i-m, j-n)
$$

where $m * n$ is the size of the convolution matrix, $w$ and $x$ are the inputs from the previous layer, and $y$ is the output of the convolutional layer.

Table 2 The configurations of the MRls database in the training and testing datasets used in tumour detection phase

\begin{tabular}{llllll}
\hline \multirow{2}{*}{ Total images } & \multicolumn{2}{l}{ Distribution in the training set } & & \multicolumn{3}{l}{ Distribution in the testing set } \\
\cline { 2 - 3 } & Normal & Abnormal & & Normal & Abnormal \\
\hline 349 & 45 & 77 & 64 & 163 \\
\hline
\end{tabular}




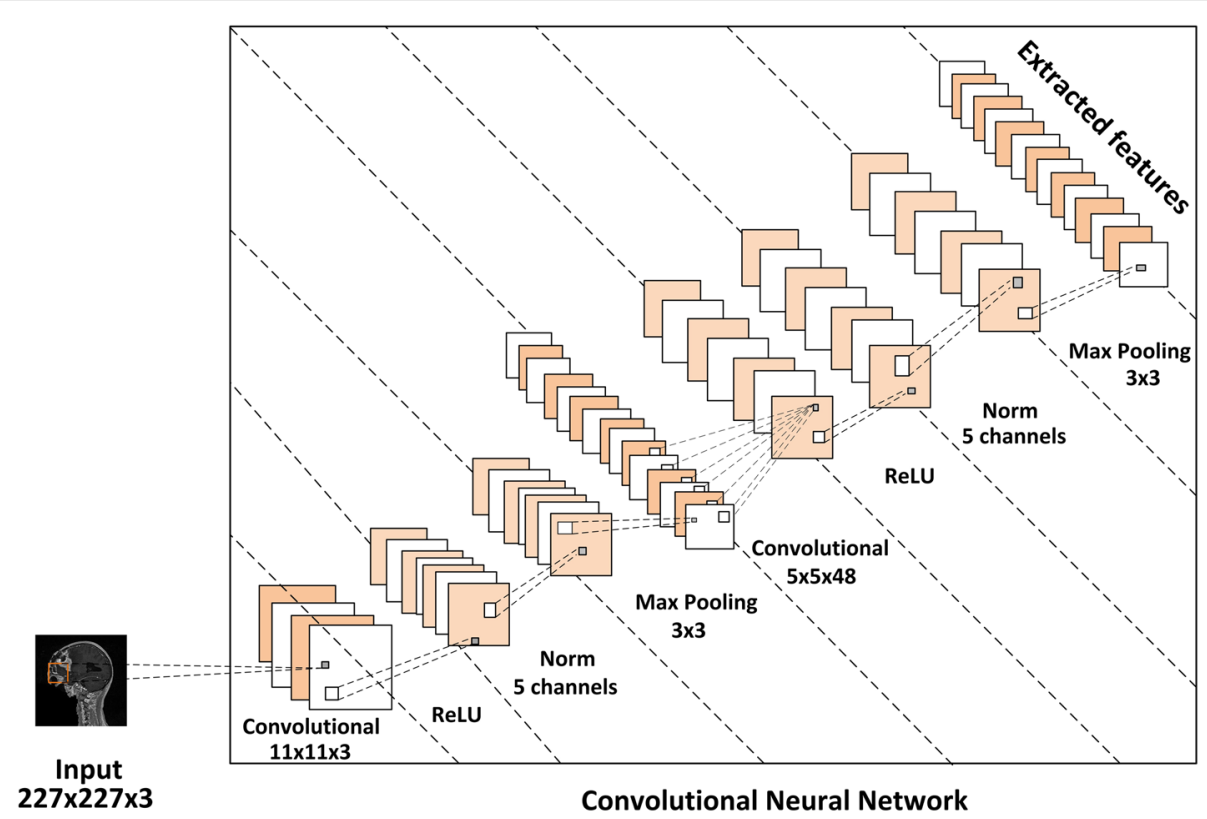

Fig. 2 The architecture of the CNN used in this study. The name of each network layer is provided. The figure shows a snapshot of the whole network architecture

To facilitate the convolution process, the dimensions of the convolutional kernel and the CNN input should be matched. If the CNN is a multidimensional array, then the kernel should be a multidimensional array as well. If the CNN input is a two-dimensional image [38], a two-dimensional kernel $w$ is used, as presented in Eq. 1.

The output of the convolutional layers, i.e., layers 2 and 6, will apply a sigmoidal activation function to the obtained convolutional output $y$ in Eq. 1 to provide the new output $f$ modelled as function of the input $y$ with Eqs. 2 or 3:

$$
\begin{aligned}
& f(y)=\tanh (y) \\
& f(y)=\frac{1}{1+e^{-y}}
\end{aligned}
$$

The output of the ReLU layers, i.e., layers 3 and 7, will apply a linear activation function to the neuron output $y$ as shown in Eq. 4:

$$
f(y)=\max (0, y)
$$

Layers 4 and 8 are the cross-channel normalization layers with five channels. The $3 \times 3$ max-pooling layers are layers 5 and 9 . These layers are very simple because they take a $K \times K$ region and output a single value, and then the output is an $\frac{N}{K} \times \frac{N}{K}$ layer. Layer 9 provides the extracted features in a dimension of $1 \times 43264$, which are then fed to the SVM to carry out the image classification.

\subsubsection{Error-correcting output codes support vector machines (ECOC-SVMs)}

Dietterich and Bakiri presented an error-correcting output codes (ECOC) approach to correct the data error when input enters into a channel. The approach uses error-correcting codes to convert the binary classifiers to a multi-class classification [39]. It has been successfully used in many applications, such as face recognition [40], face verification [41], text recognition [42], and digital manuscript classification [43].

Error-correcting output codes support vector machines (ECOC-SVMs) is a method that combines the SVMs and ECOC. It was proposed to solve the online identification and feature extraction problems. When applying ECOCSVMs to multi-classification, the $k$ classes classification is transformed to the $L$ classes classification by the ECOC matrix. ECOC-SVM functions according to the following steps [44]:

1 Training: The classes are represented in two subsets ( 0 or 1 ) for each row of the coding matrix using the SVM binary partition. The SVM takes the row with a value of 0 as the first class and the row with a value of 1 as the second class. The corresponding class assigns a codeword for each column of the matrix. All of the $L$ classes are trained according to rows of the ECOC matrix, where $L$ is the dimensionality of the new feature space and the length of the codeword.

2 Testing: The test data is fed into the learned classifier. The $L$ classifiers recognize the samples and generate output vector $Z$. The hamming distance 

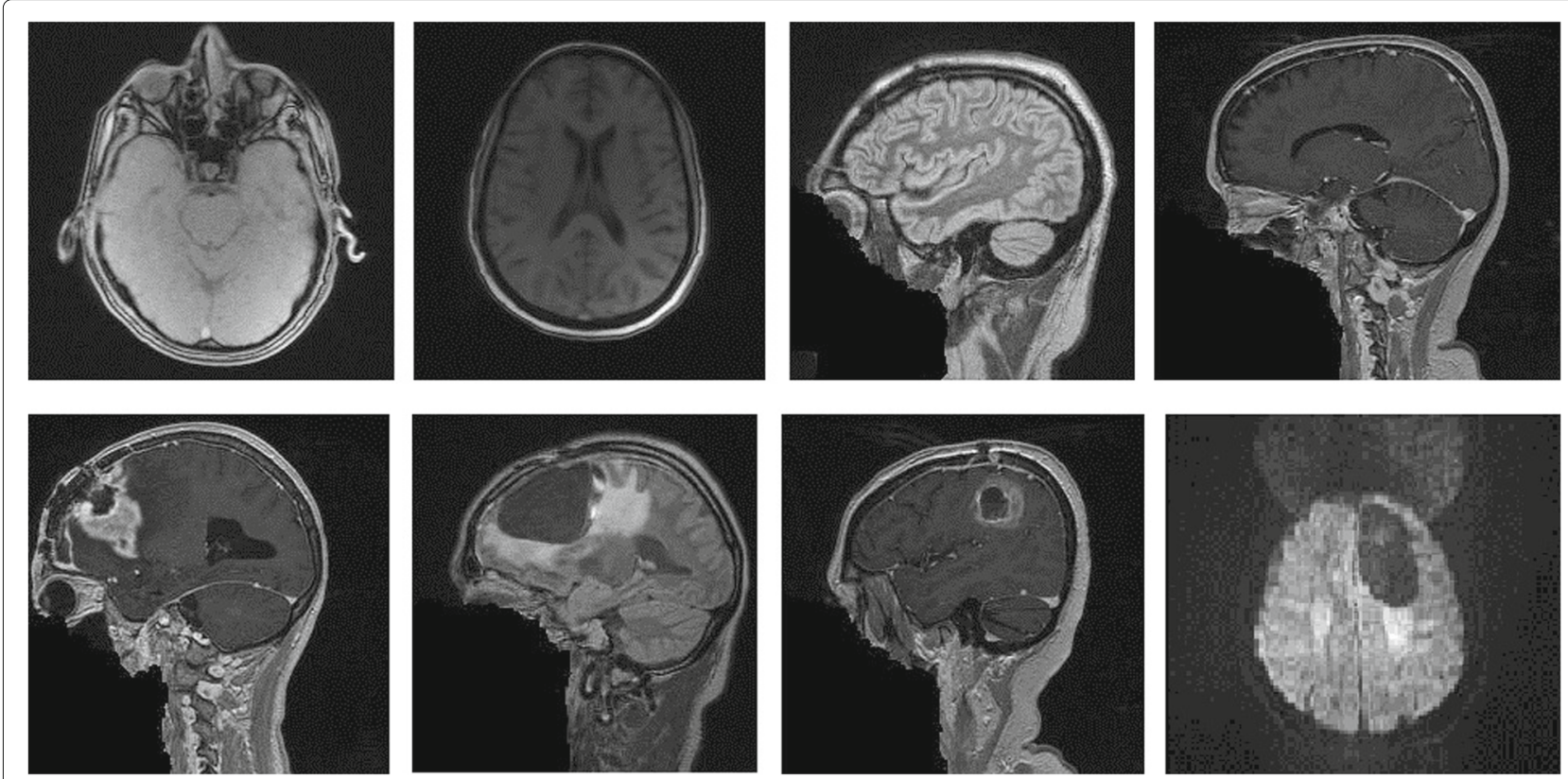

Fig. 3 Representative images from the MRIs database that was used in the tumour detection phase. Normal MRIs without brain tumours are in the top row, while abnormal images with brain tumours present are in the bottom row

between each vector in the output vector matrix and code matrix is shown in Eq. 5. The classifier chooses the class depending on the minimal distance.

$$
c_{i}=\operatorname{argmind}\left(Z, H_{i}\right)=\sum_{j=1}^{L}\left|Z_{j}-H_{i, j}\right|, i=1,2, \ldots, k
$$

Table 3 The obtained number of features, the consumed feature extraction time, and the accuracy of the different CNN layers

\begin{tabular}{llll}
\hline \multirow{2}{*}{ Layer number } & \multicolumn{3}{l}{ Evaluation metrics } \\
\cline { 2 - 4 } & No. of features & Feature extraction time & Accuracy (\%) \\
\hline Layer 2 (conv) & 290400 & 0.24 & 69.16 \\
Layer 3 (ReLU) & 290400 & 0.25 & 84.58 \\
Layer 4 (norm) & 290400 & 0.33 & 68.28 \\
Layer 5 (pool) & 69984 & 0.35 & 66.96 \\
Layer 6 (conv) & 186624 & 0.78 & 69.16 \\
Layer 7 (ReLU) & 186624 & 0.80 & 48.01 \\
Layer 8 (norm) & 186624 & 0.83 & 77.97 \\
Layer 9 (pool) & 43264 & 0.85 & 99.55 \\
Layer 10 (conv) & 64896 & 1.20 & 49.33 \\
Layer 11 (ReLU) & 64896 & 1.21 & 81.93 \\
Layer 12 (conv) & 64896 & 1.40 & 65.63 \\
Layer 13 (ReLU) & 64896 & 1.41 & 98.23 \\
Layer 14 (conv) & 43264 & 1.59 & 88.54 \\
Layer 15 (ReLU) & 43264 & 1.60 & 96.47 \\
\hline
\end{tabular}

The table indicates that the optimum number of layers with respect to the consumed time and the achieved accuracy is 9 where $H$ is the code matrix with $i$ rows and $j$ columns.

\subsection{Brain tumour localization phase}

Since the BraTS 2013 database includes 3D MRIs, we used its MRIs slice by slice as inputs for the CNN. Our method handles each slice as a 2D image [29], and T2 images were selected only with segmented images. In the preprocessing stage, we detect the tumour region from the segmented image using a bounding box method. The T2 image with the tumour bounding box are provided to the R-CNN block to train the network. As a post-processing step, we measure the properties of regions in the tested image and select the region most intersected with the $\mathrm{R}-\mathrm{CNN}$ as the tumour region.

\subsubsection{5-layer region-based convolutional neural network ( $R-C N N)$}

R-CNNs offer high object detection accuracy. The R$\mathrm{CNN}$ method trains a $\mathrm{CNN}$ to classify the image regions into an object or a background category. It uses the log loss approach to fine-tune the image regions into objects. Then, an SVM approach is applied to detect objects [45].

Table 4 Comparison of the performance of the three CNN models used in the tumour detection phase

\begin{tabular}{lll}
\hline Network model & Layer number (type) & Accuracy (\%) \\
\hline AlexNet & Layer 9 (pool) & 99.55 \\
VGG-16 & Layer 32 (pool) & 92.95 \\
VGG-19 & Layer 38 (pool) & 95.15 \\
\hline
\end{tabular}


Table 5 Comparison of the obtained tumour detection accuracy from the proposed CNN-based system to previous work in which a classical approach was used with the same database as a unified benchmark

\begin{tabular}{|c|c|c|c|c|c|c|}
\hline \multirow{2}{*}{ Detection system } & \multicolumn{6}{|c|}{ Performance evaluation metrics (\%) } \\
\hline & Sensitivity & Specificity & Accuracy & BA & PPV & NPV \\
\hline Abd-Ellah et al. [18] & 83.43 & 25.00 & 66.96 & 54.21 & 73.91 & 37.20 \\
\hline Proposed method & 99.38 & 100.0 & 99.55 & 99.69 & 100 & 98.46 \\
\hline
\end{tabular}

The R-CNN architecture used in the tumour localization phase consists of five layers. The network first processes the whole image using the image-input layer (layer 1) and then converts the input data with a size of $28 \times 28 \times 1$ into a convolutional layer (layer 2) with a filter size of 5 and filter number of 20 for detecting multiple class-specific objects. A fully connected layer (layer 3 ) with two outputs is used to predict a single-box for tumour localization. The softmax layer (layer 4) is also known as the normalized exponential. The output unit activation function is the softmax function [46-48], as presented in Eq. 6:

$$
y_{r}=\frac{\exp \left(a_{r}\right)}{\sum_{j=1}^{k} \exp \left(a_{j}\right)}
$$

where $a$ is the input vector with $k$ dimensions and $y$ is the output vector with $k$ dimensions. The denominator $\sum_{j=1}^{k} \exp \left(a_{j}\right)$ normalizes the output to ensure that $\sum_{r=1}^{k} y_{r}=1$ and $0 \leq y_{r} \leq 1$, which can be represented graphically as a layer with $k$ neurons.

The classification layer used as the final layer is named after the loss used for the training process. It returns a neural network classification output. For multi-class classification, the loss (error) function is the cross entropy function shown in Eq. 7:

$$
E(\theta)=-\sum_{i=1}^{n} \sum_{j=1}^{k} t_{i j} \ln y_{j}\left(x_{i}, \theta\right)
$$

where $\theta$ is the parameter vector, $t_{i j}$ is the indicator that the $i$ sample belongs to the $j$ class, and $y_{j}\left(x_{i}, \theta\right)$ is the output for sample $i$.

Table 6 Comparison of the proposed CNN-based brain tumour detection approach against some former state-of-the-art methods

\begin{tabular}{llll}
\hline CAD system & Total images & Classification method & Accuracy (\%) \\
\hline Da et al. [26] & 10 & DNN & 83.0 \\
Gao et al. [27] & 285 & 2D and 3D CNNs & 87.6 \\
Yan Xu et al. [28] & 45 & CNNs & 97.50 \\
Proposed method & 349 & ECOC-SVM & 99.55
\end{tabular}

\section{Results and discussion}

\subsection{Experimental environment setup}

The image database used for evaluating the tumour detection phase was extracted from the RIDER Neuro MRI database [17]. The input database consists of brain MRIs from 19 patients. These images were obtained approximately 2 days apart. T1-weighted and T2-weighted MRIs were used. The database contains a total of 349 MRIs, including 109 normal images and 240 abnormal images. A set of 43264 features was extracted from each MRI image which later was used to train the SVM. The SVM was trained using $122 \times 43264$ features in total. The specifications of the utilized database are presented in Table 2 . A sample of the MRIs database used in the experimental work is shown in Fig. 3.

To evaluate the tumour localization phase, a dataset of 804 3D MRIs extracted from the BraTS 2013 database was used for the localization accuracy assessment. The database consists of 20 high-grade (HG) and 10 low-grade (LG) patients and T1, T1c, T2, and FLAIR type MRI modalities. A set of 593 MRIs was used for R-CNN training and validation, and a set of 211 MRIs was used for the testing dataset. The 3D images were converted into 2D slices, and the T2 MRI modality was used.

\subsection{Accuracy evaluation criteria}

To evaluate the two system phases, the following standard metrics were used: sensitivity, which represents the proportion of actual positives that are correctly classified; specificity, which indicates the proportion of negatives

Table 7 Performance analysis of the developed localization R-CNN-based method compared to other methods in the literature

\begin{tabular}{lllll}
\hline \multirow{2}{*}{ Localization method } & \multicolumn{4}{l}{ Performance evaluation metrics (\%) } \\
\cline { 2 - 5 } & Layers & Sensitivity & PPV & DICE \\
\hline Havaei et al. [29] & 10 & 87.00 & 88.00 & 0.88 \\
Chang [30] & 22 & - & - & 0.87 \\
Casamitjana et al. [31] & 22 & 83.08 & 84.60 & 0.84 \\
Zhao et al. [32] & 16 & 83.00 & 92.00 & 0.87 \\
Pereira et al. [33] & 51 & 93.00 & 80.00 & 0.85 \\
Pereira et al. [34] & 11 & 89.00 & 88.00 & 0.83 \\
Proposed method & 05 & 95.44 & 81.80 & 0.87 \\
\hline
\end{tabular}


that are correctly classified; and accuracy, which is the proportion of both true positives and true negatives. These three metrics were calculated using Eqs. 8, 9, and 10 , respectively $[18,19]$ :

$$
\begin{aligned}
& \text { Sensitivity }=\frac{\sigma}{\sigma+\Phi} \\
& \text { Specificity }=\frac{\eta}{\eta+\Psi} \\
& \text { Accuracy }=\frac{\sigma+\eta}{\sigma+\eta+\Phi+\Psi}
\end{aligned}
$$

where true positives $(\sigma)$ are the correctly classified positive cases, true negatives $(\eta)$ are the correctly classified negative cases, false positives $(\Psi)$ are the incorrectly classified positive cases, and false negatives $(\Phi)$ are the incorrectly classified negative cases.

In addition to sensitivity, accuracy, and specificity, other parameters have been considered for evaluating tumour detection and localization phases. The new parameters were balanced accuracy $(\mathrm{BA})=($ sensitivity + specificity) $/ 2$, positive predictive value $(\mathrm{PPV})=\sigma /(\sigma+\Psi)$, and negative predictive value (NPV) $=\eta /(\eta+\Phi)$ [11]. Additionally, DICE scores are considered a common parameter for tumour localization approaches.

\subsection{Tumour detection accuracy analysis}

The performance of the $\mathrm{CNN}$ is measured in every network layer. The goal of this step is to identify the optimum number of network layers for achieving the highest accuracy and the shortest feature extraction time. The measured performance of each layer is recorded in Table 3. It is demonstrated in Table 3 that setting the number of layers equal to 9 provided the best performance.

Table 4 shows the best accuracy obtained from the three CNN models used in this study and the corresponding layer number. The network size has been optimized to give the best performance in learning phase and then has been fixed, with the validation and testing phases using the same network size as the learning phase. This is to generalize the $\mathrm{CNN}$ model for all data samples.

To further investigate the performance of classical learning models against the proposed deep CNN-based model, a classical brain tumour diagnosis method has been applied and studied using the same database configurations as in Table 2. The proposed CNN system achieved $99.55 \%$ accuracy, while the classical method accomplished $66.96 \%$. A short comparison between the performance of the previous classical method and the newly proposed deep learning system using the same database is presented in Table 5 . The obtained results in Table 5 show the superiority of the deep learning method in terms of brain tumour detection accuracy, which is reflected in the MRI classification.

The proposed CNN-based system has achieved $99.55 \%$ accuracy for tumour diagnosis when selecting nine CNN layers. To ensure the credibility of the obtained results,
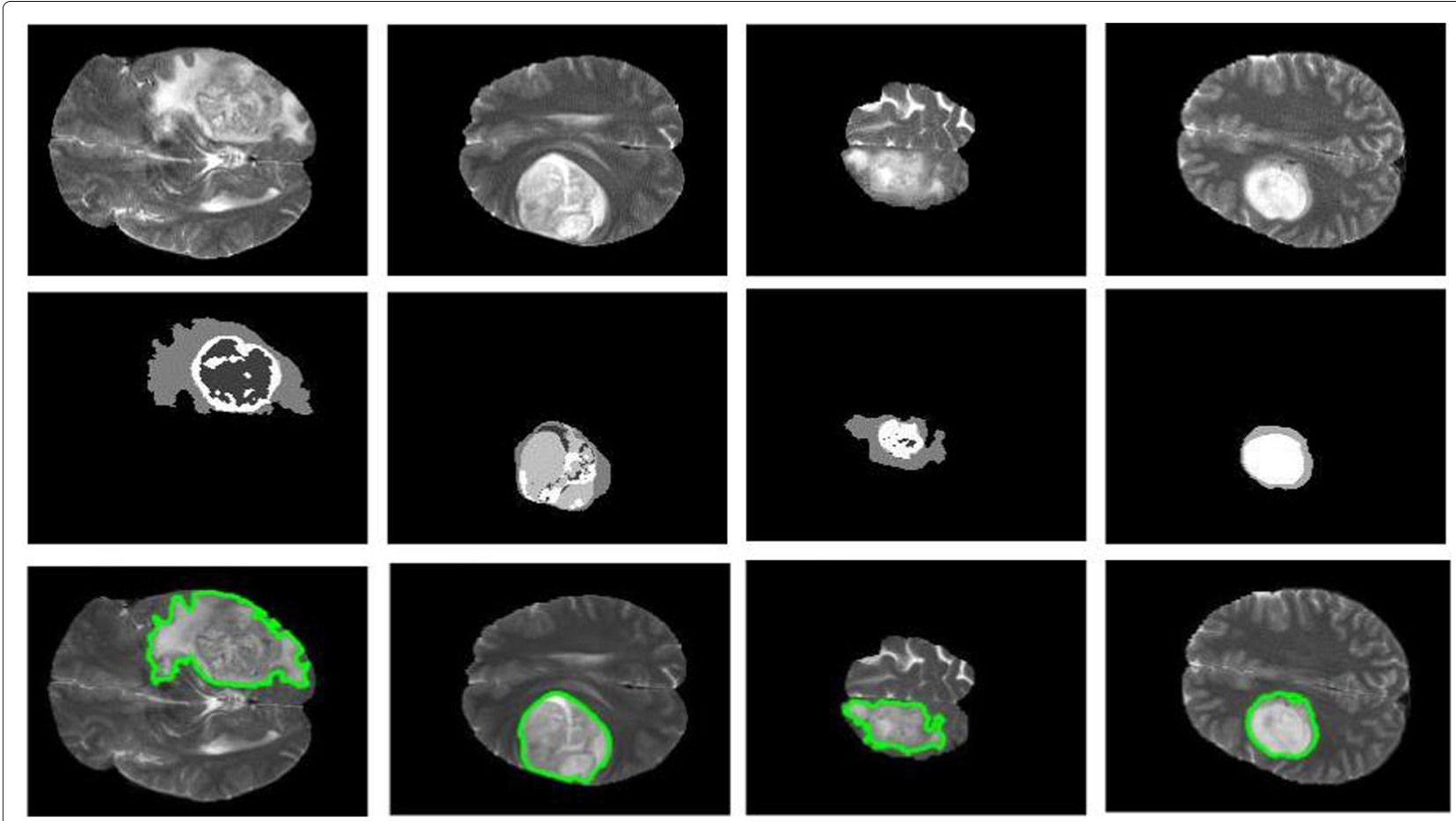

Fig. 4 Visual results in the axial view from the localization phase. The top row shows the T2 modality. The second row shows the ground truth. The bottom row shows the segmented images 
a comparison with results from the literature was performed. The outcome of the proposed CNN-based system compared with those of three popular methods reported in the literature is reported in Table 6.

\subsection{Tumour localization accuracy analysis}

Simulation experiments have been conducted to show the performance of the proposed system in fulfiling the tumour localization task. A comparison with other studies found in the literature is shown in Table 7 . It is clear that the proposed system is superior to the others in terms of the network size, sensitivity and DICE metrics but not the PPV metric. A sample of visual results from the MRIs database used in the localization phase of the experimental work is shown in Fig. 4.

\section{Conclusions}

This paper has proposed a two-phase multi-model deep learning-based system for brain tumour detection and localization from MRIs. The main goals of this study are to classify MRIs into normal and abnormal images according to the absence or presence of a brain tumour and to accurately localize the tumour within the abnormal MRIs. The first system phase employed CNN and ECOCSVM approaches for feature extraction and classification, respectively. A five-layer $\mathrm{R}-\mathrm{CNN}$ was used for tumour localization in the second system phase. The tumour detection phase was evaluated using 349 MRIs extracted from the RIDER Neuro MRI database. The empirical work has shown that the method achieved an accuracy of $99.55 \%$. The tumour localization phase was evaluated using the BraTS 2013 database, and a DICE score of 0.87 has been achieved using 804 3D MRIs. The accomplished results proved the superiority of the proposed deep learning-based method for tumour detection and demonstrate the superiority of the whole system's performance concerning both tumour detection and localization measures.

\section{Abbreviations \\ ANN: Artificial neural network; BPN: Back propagation neural network; BraTS: Brain tumour segmentation; CAD: Computer-aided diagnosis; CNN: Convolutional neural network; DNN: Deep neural network; DWTs: Discrete wavelet transforms; ECOC: Error-correcting output codes; ECOC-SVM: Error-correcting output codes support vector machine; FCM: Fuzzy C-means; FCNN: Fully convolutional neural network; FCR-NN: Fully convolutional residual neural network; GLCM: Gray-level co-occurrence matrix; HADA: Hybrid abnormality detection algorithm; HG: High grade; ICA: Independent component analysis; k-NN: K-nearest neighbours; KSVM: Kernel support vector machines; LG: Low grade; MRIs: Magnetic resonance images; NPV: Negative predictive value; PCA: Principal component analysis; PNN: Probabilistic neural network; PPV: Positive predictive value; RBFN: Radial basis function neural network; R-CNN: Region-based convolutional neural network; ReLU: Rectified linear unit; RIDER: Reference Image Database to Evaluate Response; SOMNN: Self organizing map neural network; VGG: Visual Geometry Group.}

\section{Acknowledgements}

The authors are very thankful to the editor and the anonymous reviewers for their valuable comments and suggestions.

\section{Availability of data and materials}

Not applicable. The magnetic resonance images used in this study are public databases that are cited within the text.

\section{Authors' contributions}

MA was the main contributor to the ANNs design, implementation, and evaluation. He worked on the MRI databases preparation and the manuscript writing as well. AA focused on the technical results and developed comparisons with the state-of-the-art. He also added valuable efforts into preparing the manuscript. AK worked on the mathematical models of ANNs and offered help in the empirical work done. He also provided a strong support to the manuscript writing process. HH supervised the research outcomes and gave help in the manuscript writing and editing processes. $\mathrm{HH}$ provided feedback on the whole structure of the manuscript as well. All authors read and approved the final manuscript.

\section{Ethics approval and consent to participate}

Not applicable.

\section{Competing interests}

The authors declare that they have no competing interests.

\section{Publisher's Note}

Springer Nature remains neutral with regard to jurisdictional claims in published maps and institutional affiliations.

\section{Author details}

${ }^{1}$ Electronic and Communication Department, Al-Madina Higher Institute for Engineering and Technology, Giza, Egypt. ${ }^{2}$ Department of Computer Science, Electrical and Space Engineering, Luleå University of Technology, SE-97187 Luleå, Sweden. ${ }^{3}$ Faculty of Engineering, Al-Azhar University, P.O. Box 83513, Qena, Egypt. ${ }^{4}$ Faculty of Engineering, Minia University, Minia, Egypt, P.O. Box 61111, Minia, Egypt.

Received: 19 September 2017 Accepted: 7 September 2018

Published online: 30 September 2018

\section{References}

1. T. Logeswari, M. Karnan, An improved implementation of brain tumor detection using segmentation based on hierarchical self organizing map. Int. J. Comput. Theory Eng. 2(4), 591 (2010)

2. E.-S. A. El-Dahshan, H. M. Mohsen, K. Revett, A.-B. M. Salem, Computer-aided diagnosis of human brain tumor through MRI: A survey and a new algorithm. Expert Syst. Appl. 41(11), 5526-5545 (2014)

3. D. Jayadevappa, S. Srinivas Kumar, D. Murty, Medical image segmentation algorithms using deformable models: a review. IETE Tech. Rev. 28(3), 248-255 (2011)

4. S. Yazdani, R. Yusof, A. Karimian, M. Pashna, A. Hematian, Image segmentation methods and applications in MRI brain images. IETE Tech. Rev. 32(6), 413-427 (2015)

5. L. Zhao, K. Jia, Multiscale CNNs for brain tumor segmentation and diagnosis. Comput. Math. Methods Med. 2016, 7 (2016). https://doi.org/ 10.1155/2016/8356294. Article ID 8356294

6. A. Madabhushi, G. Lee, Image analysis and machine learning in digital pathology: challenges and opportunities. Med. Image Anal. 33, 170-175 (2016). 20th anniversary of the Medical Image Analysis journal (MedIA)

7. J. Jiang, P. Trundle, J. Ren, Medical image analysis with artificial neural networks. Comput. Med. Imaging Graph. 34(8), 617-631 (2010)

8. D. J. Hemanth, C. K. S. Vijila, A. I. Selvakumar, J. Anitha, Performance improved iteration-free artificial neural networks for abnormal magnetic resonance brain image classification. Neurocomputing. 130, 98-107 (2014)

9. G. E. Hinton, S. Osindero, Y.-W. Teh, A fast learning algorithm for deep belief nets. Neural Comput. 18(7), 1527-1554 (2006)

10. C. Yan, F. Coenen, B. Zhang, Driving posture recognition by convolutional neural networks. IET Comp. Vision. 10, 103-11411 (2016)

11. H.-I. Suk, S.-W. Lee, D. Shen, Deep ensemble learning of sparse regression models for brain disease diagnosis. Med. Image Anal. 37, 101-113 (2017)

12. A. Krizhevsky, I. Sutskever, G. E. Hinton, in Proceedings of the 25 th International Conference on Neural Information Processing Systems - Volume 1. 
ImageNet Classification with Deep Convolutional Neural Networks (Curran Associates Inc., Lake Tahoe, Nevada, 2012), pp. 1097-1105

13. C. Yan, H. Xie, D. Yang, J. Yin, Y. Zhang, Q. Dai, Supervised hash coding with deep neural network for environment perception of intelligent vehicles. IEEE Trans. Intell. Transp. Syst. 19(1), 284-295 (2018). https://doi. org/10.1109/TITS.2017.2749965

14. A. Şentaş, I. Tashiev, F. Küçükayvaz, S. Kul, S. Eken, A. Sayar, Y. Becerikli, in Advances in Internet, Data \& Web Technologies, ed. by L. Barolli, F. Xhafa, N. Javaid, E. Spaho, and V. Kolici. Performance evaluation of support vector machine and convolutional neural network algorithms in real-time vehicle type classification (Springer, Cham, 2018), pp. 934-943

15. Y. LeCun, K. Kavukcuoglu, C. Farabet, in Proceedings of 2010 IEEE International Symposium on Circuits and Systems. Convolutional networks and applications in vision (IEEE, Paris, 2010), pp. 253-256

16. J. Lei, G. Li, J. Zhang, Q. Guo, D. Tu, Continuous action segmentation and recognition using hybrid convolutional neural network-hidden Markov model model. IET Comput. Vision. 10, 537-5447 (2016)

17. The Cancer Imaging Archive, RIDER NEURO MRI Database (2016). https:// wiki.cancerimagingarchive.net/display/Public/RIDER+NEURO+MRI. Accessed 14 Sept 2018

18. M. K. Abd-Ellah, A. I. Awad, A. A. M. Khalaf, H. F. A. Hamed, in Building Sustainable Health Ecosystems: 6th International Conference on Well-Being in the Information Society, WIS 2016, Tampere, Finland, September 16-18, 2016, Proceedings, ed. by H. Li, P. Nykänen, R. Suomi, N. Wickramasinghe, G. Widén, and M. Zhan. Classification of brain tumor MRIs using a kernel support vector machine, vol. 636 (Springer, Cham, 2016), pp. 151-160

19. M. K. Abd-Ellah, A. I. Awad, A. A. M. Khalaf, H. F. A. Hamed, in IEE International Conference on Microelectronics, ICM 2016, Giza, Egypt, December 17-20, 2016. Design and implementation of a computer-aided diagnosis system for brain tumor classification (IEEE, 2016), pp. 73-76

20. C. Lakshmi Devasena, M. Hemalatha, Efficient computer aided diagnosis of abnormal parts detection in magnetic resonance images using hybrid abnormality detection algorithm. Cent. Eur. J. Comput. Sci. 3(3), 117-128 (2013)

21. S. Patil, V. R. Udupi, A computer aided diagnostic system for classification of brain tumors using texture features and probabilistic neural network. Int. J. Comput. Sci. Eng. Inf. Technol. Res. 3, 61-66 (2013)

22. M. P. Arakeri, G. R. M. Reddy, Computer-aided diagnosis system for tissue characterization of brain tumor on magnetic resonance images. Sig. Image Video Process. 9(2), 409-425 (2015)

23. E. Dandıl, M. Çakıroğlu, Z. Ekşi, in ICT Innovations 2014. Computer-aided diagnosis of malign and benign brain tumors on MR images (Springer, Cham, 2015), pp. 157-166

24. S. Goswami, L. K. P. Bhaiya, in 2013 International Conference on Communication Systems and Network Technologies. Brain tumor detection using unsupervised learning based neural network (IEEE, Gwalior, 2013), pp. $573-577$

25. S. N. Deepa, B. A. Devi, in 2012 International Conference on Computer Communication and Informatics, ICCCI-2012, 10-12 January, 2012. Artificial neural networks design for classification of brain tumour (IEEE, Coimbatore, 2012), pp. 1-6

26. C. Da, H. Zhang, Y. Sang, in Proceedings of the 18th Asia Pacific Symposium on Intelligent and Evolutionary Systems. Brain CT image classification with deep neural networks, vol. 1 (Springer International Publishing, Cham, 2015), pp. 653-662

27. X. W. Gao, R. Hui, Z. Tian, Classification of CT brain images based on deep learning networks. Comput. Methods Prog. Biomed. 138, 49-56 (2017)

28. Y. Xu, Z. Jia, Y. Ai, F. Zhang, M. Lai, E. I. Chang, in 2015 IEEEE International Conference on Acoustics, Speech and Signal Processing, ICASSP, 19-24 April, 2015. Deep convolutional activation features for large scale brain tumor histopathology image classification and segmentation (IEEE, Brisbane, 2015), pp. 947-951

29. M. Havaei, A. Davy, D. Warde-Farley, A. Biard, A. Courville, Y. Bengio, C. Pal, P.-M. Jodoin, H. Larochelle, Brain tumor segmentation with deep neural networks. Med. Image Anal. 35, 18-31 (2017)

30. P. D. Chang, in Brain lesion: glioma, multiple sclerosis, stroke and traumatic brain injuries: Second International Workshop, BrainLes 2016, with the Challenges on BRATS, ISLES and mTOP 2016, Held in Conjunction with MICCAI 2016, Athens, Greece, October 17, 2016, Revised Selected Papers, ed. by A. Crimi, B. Menze, O. Maier, M. Reyes, S. Winzeck, and H. Handels. Fully convolutional deep residual neural networks for brain tumor segmentation (Springer, Cham, 2016), pp. 108-118

31. A. Casamitjana, S. Puch, A. Aduriz, V. Vilaplana, in Brainlesion: glioma, multiple sclerosis, stroke and traumatic brain injuries: Second International Workshop, BrainLes 2016, with the Challenges on BRATS, ISLES and mTOP 2016, Held in Conjunction with MICCAI 2016, Athens, Greece, October 17, 2016, Revised Selected Papers, ed. by A. Crimi, B. Menze, O. Maier, M. Reyes, $\mathrm{S}$. Winzeck, and $\mathrm{H}$. Handels. $3 \mathrm{~d}$ convolutional neural networks for brain tumor segmentation: a comparison of multi-resolution architectures (Springer, Cham, 2016), pp. 150-161

32. X. Zhao, Y. Wu, G. Song, Z. Li, Y. Fan, Y. Zhang, in Brainlesion: glioma, multiple sclerosis, stroke and traumatic brain injuries: Second International Workshop, BrainLes 2016, with the Challenges on BRATS, ISLES and mTOP 2016, Held in Conjunction with MICCAI 2016, Athens, Greece, October 17, 2016, Revised Selected Papers, ed. by A. Crimi, B. Menze, O. Maier, M. Reyes, $\mathrm{S}$. Winzeck, and $\mathrm{H}$. Handels. Brain tumor segmentation using a fully convolutional neural network with conditional random fields (Springer, Cham, 2016), pp. 75-87

33. S. Pereira, A. Oliveira, V. Alves, C. A. Silva, in 2017 IEEE 5th Portuguese Meeting on Bioengineering (ENBENG). On hierarchical brain tumor segmentation in MRI using fully convolutional neural networks: a preliminary study, (2017), pp. 1-4. https://doi.org/10.1109/ENBENG.2017.7889452

34. S. Pereira, A. Pinto, V. Alves, C. A. Silva, Brain tumor segmentation using convolutional neural networks in MRI images. IEEE Transactions on Medical Imaging. 35(5), 1240-1251 (2016). https://doi.org/10.1109/TMI. 2016.2538465

35. A. Işin, C. Direkoğlu, M. Şah, Review of MRI-based brain tumor image segmentation using deep learning methods. Procedia Comput. Sci. 102 317-324 (2016). 12th International Conference on Application of Fuzzy Systems and Soft Computing, ICAFS 2016, 29-30 August 2016, Vienna, Austria

36. R. C. Gonzalez, R. E. Woods, Digital Image Processing, 3rd edn. (Prentice-Hall, Inc., Upper Saddle River, 2006)

37. Y. Pan, W. Huang, Z. Lin, W. Zhu, J. Zhou, J. Wong, Z. Ding, Brain tumor grading based on neural networks and convolutional neural networks. 37th Annual International Conference of the IEEE Engineering in Medicine and Biology Society, Milan, Italy, 25-29 August, EMBC, 699-702 (2015)

38. Y. B. lan Goodfellow, A. Courville, Deep learning. Book in preparation for MIT Press (2016). http://www.deeplearningbook.org. Accessed 14 Sept 2018

39. T. G. Dietterich, G. Bakiri, Solving multiclass learning problems via error-correcting output codes. J. Artif. Intell. Res. 2, 263-286 (1995)

40. T. Windeatt, G. Ardeshir, in 2003 International Conference on Visual Information Engineering, VIE 2003. Boosted ECOC ensembles for face recognition (IET, Guildford, 2003), pp. 165-168

41. J. Kittler, R. Ghaderi, T. Windeatt, J. Matas, in Proceedings of the 2001 IEEE Computer Society Conference on Computer Vision and Pattern Recognition, CVPR 2001, vol 1. Face verification using error correcting output codes, (2001), pp. 755-760

42. R. Ghani, in Proceedings of the 2001 IEEE International Conference on Data Mining. Combining labeled and unlabeled data for text classification with a large number of categories, (2001), pp. 597-598

43. J. Zhou, C. Y. Suen, in Eighth International Conference on Document Analysis and Recognition, ICDAR'05, vol 1. Unconstrained numeral pair recognition using enhanced error correcting output coding: a holistic approach, (2005), pp. 484-488

44. F. Deng, S. Guo, R. Zhou, J. Chen, Sensor multifault diagnosis with improved support vector machines. IEEE Trans. Autom. Sci. Eng. PP(99), 1-11 (2015)

45. R. Girshick, Fast R-CNN. International Conference on Computer Vision (ICCV), Santiago, Chile, 11-18 December, 2015. (IEEE, Santiago, 2015), pp. 1440-1448

46. P. Reverdy, N. E. Leonard, Parameter estimation in softmax decision-making models with linear objective functions. IEEE Trans. Autom. Sci. Eng. 13(1), 54-67 (2016)

47. C. M. Bishop, Pattern recognition and machine learning. (Springer, 2006)

48. B. Yuan, in 2016 29th IEEE International System-on-Chip Conference (SOCC). Efficient hardware architecture of softmax layer in deep neural network (IEEE, Seattle, 2016), pp. 323-326 